\title{
Ruptured Bilateral Tubo-Ovarian Abscess with Multi-Organ Failure after Sonosalpingography
}

\author{
P. Singh ${ }^{1}$, Y. Pradeep ${ }^{2}$, C. Mahajan ${ }^{3}$ \\ ${ }^{1}$ Department of Obstetrics and Gynaecology, Dr. Ram Manohar Lohia Institute of Medical Sciences, Lucknow, Uttar \\ Pradesh, India. ${ }^{2}$ Department of Obstetrics and Gynaecology, Dr. Ram Manohar Lohia Institute of Medical Sciences, \\ Lucknow, Uttar Pradesh, India. ${ }^{3}$ Department of Obstetrics and Gynaecology, Dr. Ram Manohar Lohia Institute of \\ Medical Sciences, Lucknow, Uttar Pradesh, India.
}

\section{INTRODUCTION}

Tubo ovarian abscess is an extremely rare event after sonosalpingography. TOA is a serious and potentially life-threatening condition. Aggressive medical and/or surgical therapy is required, and rupture of an abscess may result in sepsis. It is mostly associated with chronic pelvic inflammatory disease (PID), endometriosis and endometrioma and post caesarean section. The most common cause of TOA is ascending infection which is the major pathophysiology in the development of PID. Other causes of TOA are hematogenous spread, lymphatic spread from contiguous organ, infection in previously existing ovarian cyst and flare up of an old infection.(1,2) Tubo-ovarian abscess is characterized by an inflammatory complex mass involving tubes, ovaries, and other adjacent organs like bowel or bladder. The diagnosis of TOA requires a PID criteria and at least one complex pelvic mass.(4) Appropriate management is crucial, because there are potentially severe short term consequences such as abscess rupture, peritonitis and sepsis, and long term consequences such as infertility, ectopic pregnancy and chronic PID.(5) In most cases, TOA responds well to antibiotics; however, $25 \%$ cases of TOA require surgical intervention and drainage. ${ }^{(6)}$ Here we are reporting a rare case of TOA in infertile woman who underwent SSG.

\section{PRESENTATION OF CASE}

A 25-year-old woman was admitted in emergency at Department of OBGYN, Dr. RMLIMS with septicaemia following sonosalpingography (SSG). She developed high grade fever with chills, nausea, vomiting, 48 hours after the procedure. She was admitted in District Hospital and started on broad spectrum I.V. antibiotics, and analgesics. In spite of these measures, she did not respond, and her condition was deteriorating. She was referred to Dept. of OBGYN, DRRMLIMS.

She was married for 2 years and had history of dysmenorrhoea. She was under treatment for infertility. There was no known history of PID or STD. USG- bilateral endometrioma was diagnosed. She underwent SSG with saline and Cifran to determine patency of tubes. SSG shows delayed spillage with fluid in pouch of Douglas. At the time of admission, she was ill, febrile (oral temperature was 38.5 centigrade, her pulse, blood pressure, and respiratory rate were 120 /minute, 120/70,18/minutes respectively. Clinical examination- peritoneal signs \& abdominal tenderness present. On P/S and P/V, cervix was found to be congested and pulled up; frozen pelvis was noted. Lab studies show severe anaemia with haemoglobin of 6.0 gm, TLC-24000/-,with polymorphocytosis, CRP and ESR were raised, serum creatinine-3.5, serum bilirubin 1.8, s. potassium- 5.5 IU; USG shows $9.7 \times 6.0$ × $7.4 \mathrm{~cm}$ cyst with echogenic fluid in POD internal echoes vol- $230 \mathrm{cc}$ in left adnexa and $6.2 \mathrm{x}$ $3.9 \times 4.5 \mathrm{~cm}$ volume- $58 \mathrm{cc}$ cyst in right adnexa. CA-125 was slightly elevated.
Corresponding Author: Dr. Pratibha Singh, 5/161, Vinay Khand-5, Gomti Nagar, Lucknow-226010, Uttar Pradesh, India.

E-mail: drpratibhakgmc@gmail.com

DOI: $10.14260 / \mathrm{jemds} / 2019 / 740$

Financial or Other Competing Interests: None.

How to Cite This Article:

Singh P, Pradeep Y, Mahajan C. Ruptured bilateral tubo-ovarian abscess with multiorgan failure after sonosalpingography.. J. Evolution Med. Dent. Sci. 2019;8(45):34133415, DOI: $10.14260 /$ jemds/2019/740

Submission 10-09-2019,

Peer Review 23-10-2019,

Acceptance 30-10-2019,

Published 11-11-2019. 


\section{DISCUSSION OF MANAGEMENT}

Patient is stabilized, two units of PRBC were transfused to the patient. After taking a vaginal swab for $\mathrm{c} / \mathrm{s}$, intravenous antibiotics Imipenem $500 \mathrm{mg}$ BD, inj. Gentamycin 3-5 mg TDS, were started. Decision of laparotomy was taken. Pus was present in peritoneal cavity. There was a large ruptured tuboovarian abscess arising from right adnexa. Distorted anatomy, severe adhesions to uterus, colon and small intestines were seen. Approximately $300 \mathrm{cc}$ of pus was drained. Adhesiolysis was followed by removal of abscess cavity and associated necrotic tissue fluid and debris. Abdomen was closed after peritoneal lavage and drainage. During the post-op period, patient was still having a temperature of $38^{\circ} \mathrm{C}$. Pus culture showed E.coli sensitive to meropenem and imipenem. She was already on Imipenem. The dose was escalated to $500 \mathrm{mg}$ TDS. Patient responded, gradually improved and was discharged on $7^{\text {th }}$ post op day. Patient was well without recurrence at 3 months follow up. The final histopathologic findings were- a bilateral ovarian cyst with fallopian tube; acute on chronic salpingitis with chronic inflammatory pathology of ovary.

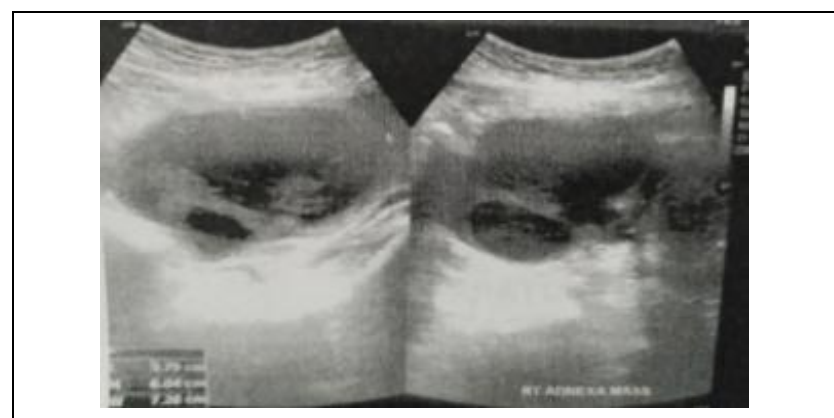

Figure 1. Ultrasound of the Patient Revealing Right Adnexal Mass Measuring $9.79 \mathrm{~cm}$ x $6.04 \mathrm{~cm} \times 7.3 \mathrm{~cm}$, Volume-Approx. $228.52 \mathrm{ml}$.

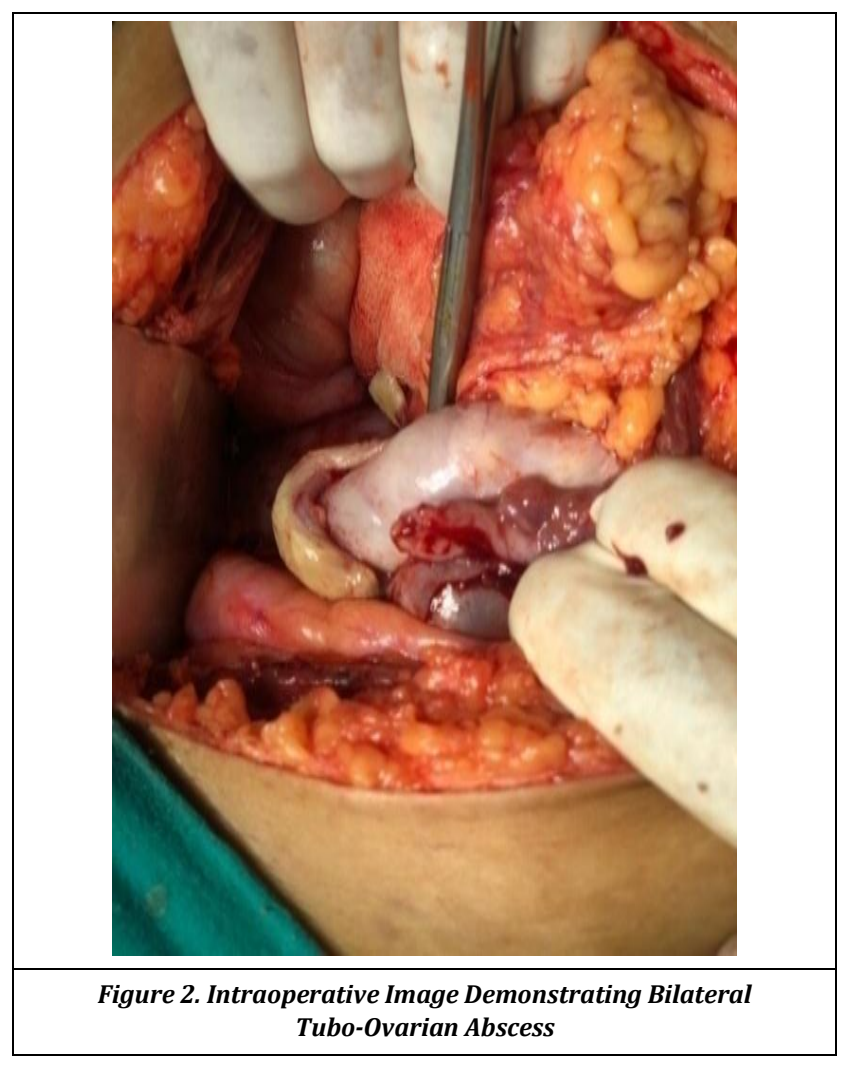

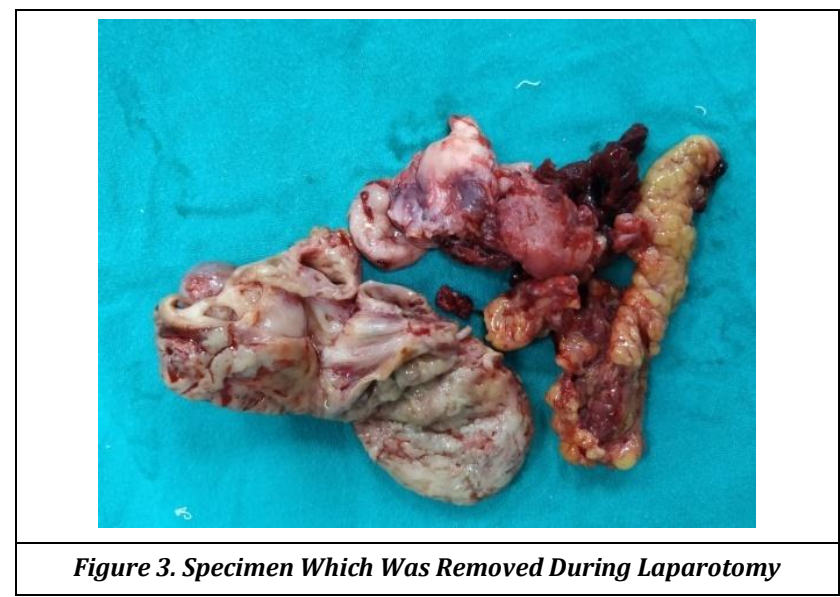

DISCUSSION

Pelvic inflammatory diseases (PID) is an infection and inflammatory disorder of upper female reproductive tract (the uterus, fallopian tube, and adjacent pelvic structures). It is initiated by infection which ascends from vagina and cervix. Chlamydia trachomatis is the predominant sexually transmitted organism causing PID. Recent studies shown that PID may often polymicrobial in nature (30-40\%).Other organism that have been implicated in pathogenesis of PID include Neisseria gonorrhoeae, Hemophilus influenza, Gardnerella vaginalis, and anaerobes such as Peptococcus and Bacteroides species. Tubo-ovarian abscess is collection of pus involving the ovary and fallopian tube (Distal part). TOA most often occurs as a consequence of PID. TOA rarely can occur following Sonosalpingography. TOA involvement of an endometrioma has been reported in cases of patients with polymicrobial sources but Kavoussi et al(7) reported a case of endometrioma complicated by TOA in patient of bacterial vaginosis (BV).They concluded aggressive treatment of bacterial vaginosis in patient with known advanced stage of endometriosis may be considered to prevent super infected endometriomas.(8)The development of TOA among women with endometriomas may be due to increased susceptibility to infection, particularly in altered immune environment seen with ectopic endometrial glands and stroma, although there is no epidemiologic data to support this theory. ${ }^{(6,8)}$ Previous surgical procedure have been found to be increased risk of TOA formation in patient of endometriosis.(4)Response of Medical management is good for un-ruptured abscess less than seven $\mathrm{cm}$ in diameter.(9) Ruptured Tubo-ovarian abscess require an aggressive approach in order to prevent mortality and morbidity. Four principle indication for laparotomy, laparoscopy are 1-suspicion of surgical emergency (Rupture of the abscess or organs) 2. Unsuccessful drainage of an abscess. 3. Poor response to treatment and drainage and antibiotics, 4. Uncertainty about the diagnosis.(4)

In this case the SSG was done, despite the presence of endometrioma. We conclude that TOA with septicaemia with multi organ failure could have been avoided by taking care of endometrioma; prior investigation to rule out PID and Bacterial Vaginosis. High index of suspicion for early diagnosis and prompt management with appropriate antibiotics in full doses. 


\section{FINAL DIAGNOSIS}

Post SSG ruptured TOA in septicaemia with multiorgan failure (SOFA score 10). Acute on chronic salpingitis with chronic inflammatory pathology of ovary.

\section{REFERENCES}

[1] Han C, Wang C, Liu XJ, et al. In vitro fertilization complicated by rupture tubo-ovarian abscess during pregnancy. Taiwan J Obstet Gynecol 2015;54(5):612-6.

[2] Pabuccu EG, Taskin S, Atabekoglu C, et al. Early pregnancy loss following laparoscopic management of ovarian abscess secondary to oocyte retrievel. Int J Fertil Steril 2014;8(3):341-6.

[3] Landers DV, Sweet RL. Tubo-ovarian abscess: contemporary approach to management. Rev Infect Dis 1983;5(5):876-84.
[4] Department of Health and Human Services: Centre for disease control and prevention: Pelvic Inflammatory disease. MMWR 2010;59(RR-12)63-7.

[5] To V, Gurberg J. Krishnamurthy S. Tubo-Ovarian abscess caused by Candida Albicans in obese patient. J Obstet Gynecol Can 2015;37(5);426-9.

[6] Granberg S, Gjelland K, Ekerhovd E. The management of pelvic abscess. Best Pract Res Clin Obstet Gynecol 2009;23(5):667-78.

[7] Kavoussi SK, Pearlman MD, Burke WM, et al. Endometrioma complicated by tubo-ovarian abscess in a woman with bacterial vaginosis. Infect Dis Obstet Gynecol 2006;2006:84140.

[8] Kitamura S, Matsumura N, Ohtake N, et al. Tubo-ovarian abscess with endometrial cyst probably infected by Campylobacter fetus: two cases. J Obstet Gynecol Res 2016;42(8);1052-7.

[9] Beigi RH, Sharp HT, Eckler K. Management and complication of tubo-ovarian abscess. Up To Date 2019. www.uptodate.com. 